\title{
Gestão estratégica do conhecimento no campo da avaliação em educação a distância
}

\author{
Eleonora Milano Falcão Vieira* \\ Neri dos Santos ${ }^{\dagger \dagger}$
}

\section{Resumo}

A gestão estratégica do conhecimento aplicada ao campo da avaliação institucional visa, principalmente, a construção de um sistema informático que permita mensurar a adequação de desempenho dos diversos processos que compõe 0 Ensino a Distância.

A modelagem a ser desenvolvida é baseada nas demandas das organizações públicas envolvidas e no levantamento de requisitos realizado junto às equipes multidisciplinares que trabalham com educação a distância em Instituições Federais de Ensino Superior. A identificação dos requisitos de informação dos agentes envolvidos permitirá a proposição de uma ferramenta de avaliação integrada e abrangente, que possa proporcionar uma retroalimentação de informações confiáveis e eficientes.

Palavras-chaves: conhecimento; gestão; avaliação; educação a distância.

\begin{abstract}
Applied to institucional evaluation strategic knowledge management, the buliding of a system that allows weight the performance of the complexity processes that Distance Distance Education.

The systems modeling to be developed is based on the demands of the public organizations envolved and the survey of requirements made with the multidisciplinary groups working with Distance Education at the Federal Institutions of Higher Education. The identification of the information needs of the stakeholders will allows the proposal of a evaluation tool integrated and widespread, able to result in a trustful and eficient information.
\end{abstract}

Key Words: knowledge; evaluation, distance education, management

\section{Introdução}

O conhecimento e a informação são as forças mais imponentes da nova época, a era informacional como a definiu Castells (1999). É preciso, no entanto, ter presente as concepções do conhecimento, decisivas para se obter as melhores e mais atuais estratégias nas organizações. Primeiramente, o conhecimento como nova formulação teórica sobre fenômenos da natureza e da sociedade; conjunto de novas idéias, de técnicas, de descobertas científicas. O conhecimento é sempre o novo, o que provoca mudança e transformação. Dada a capacidade da mente humana de permanentemente criar novas formas de conhecimento, a sociedade evolui, inovando seus métodos de ação. Essa é uma necessidade imperiosa a todas as formas de organização e, muito particularmente, aquelas voltadas para o processo educacional.

Nas duas últimas décadas tem crescido o debate e a preocupação com o desempenho de instituições educacionais no contexto internacional, especialmente, quando muitos governos iniciam um processo de

\footnotetext{
* Titulação: Doutoranda do Programa de Pós-Graduação em Engenharia e Gestão do Conhecimento e Professora do Departamento de Ciências Contábeis da Universidade Federal de Santa Catarina. E-mail: emfv0006@ yahoo.com.br. Endereço: Universidade Federal de Santa Catarina - Centro Sócio-Econômico Departamento de Ciências Contábeis - Campus Universitário Trindade - CEP 88040-970.

${ }^{\dagger}$ Pós- Doutor pela Ecole Polytechnique de Montreal, EPM, Canadá. Professor do Departamento de Engenharia de Produção da Universidade Federal de Santa Catarina. E-mail: neri@ deps.ufsc.br. Endereço: Universidade Federal de de Santa Catarina - Programa de Pós-Graduação em Engenharia e Gestão do Conhecimento - Campus Universitário Trindade - CEP 88040-970.

Artigo recebido em maio de 2005 e aceito para publicação em junho de 2005.
} 
desregulamentação dos cursos de graduação e pós-graduação e quando há um considerável aumento na oferta desses cursos à distância (Gilroy et al., 2001; Pounder, 1999; Martens e Prosser, 1998; Varey, 1993).

É possível concluir, a partir da literatura consultada, que a adoção de práticas de controle e garantia de qualidade nas instituições de ensino superior especialmente naquelas que já oferecem cursos ou pretendem oferecer cursos a distância, torna-se obrigatória. Esta exigência tem levado à criação de diretrizes e critérios para garantir a qualidade na oferta de cursos. Devido às imposições colocadas pelo aumento da competição decorrente da globalização da oferta de serviços educativos, juntamente com as exigências das agências reguladoras e/ou certificadoras, mais imperiosa torna-se a adoção de mecanismos avaliativos capazes de assegurar a qualidade dos cursos.

Dessa forma Tait (apud Moraes, 2004, p. 29) destaca a importância do contexto para qualquer iniciativa e que ao definir sistemas para a garantia de qualidade esses devem buscar a definição de seus objetivos em conjunto com seus usuários ou com pleno conhecimento das necessidades deles, uma vez que ao garantir qualidade busca-se a satisfação do usuário ou cliente, ou seja, o consumidor dos serviços.

Nesse sentido, e resguardada a natureza do serviço público no setor educacional, a implantação de um sistema de informatização para avaliação de cursos oferecidos a distância se torna recorrente aos instrumentos de modernização de práticas, já disponíveis e aplicados em setores da atividade privada. Nesse sentido, o conhecimento torna-se o instrumento fundamental à gestão estratégica de formas educacionais novas, capazes de atender às exigências de uma época de mudanças e inovações. A educação a distância é um desses campos educacionais suscetíveis de aplicação do conhecimento como estratégia de gestão, principalmente, no uso adequado da informação.

Segundo Heemann (1997, p.2) "informação poderia ser vista como um conjunto de dados relacionados com a finalidade de transmitir conhecimento". A autora cita três tipologias da informação: informação estratégica, informação operacional e informação corrente.

A informação estratégica tem um âmbito mais extenso e profundo, pois possibilita a estratégia de ação para determinada situação e o planejamento estratégico projetado, no tempo, como iniciativa institucional. Essa é a informação do sistema da organização, e que permite a um processo acadêmico de credenciamento, autorização ou reconhecimento de cursos, por exemplo, se deslocar, tecnicamente, ao longo da estrutura, até atingir seu objetivo. Esse deslocamento deve ser caracterizado por um trânsito nas redes logísticas do sistema, de forma eficiente, racional e ágil.

Nestas tipologias da informação é preciso destacar a importância vital da qualidade, expressa por Heemann (1997, p.5 ): "o padrão de qualidade representa o grau de aderência ou conformidade esperado de um processo, produto ou serviço, em relação aos requisitos e expectativas - reais e potenciais - dos clientes".

O conhecimento, o uso das tecnologias de informação e a comunicação são forças primordiais da educação a distância. A qualidade e a acessibilidade das informações vão garantir a agilidade dos processos e conseqüentemente o sucesso dos cursos oferecidos a distância. Portanto, o cenário que se descortina é o de uma qualificada gestão estratégica do conhecimento, no qual se integram processos de aprendizagem, informação, avaliação e competências.

\section{Conhecimento e gestão estratégica}

O conhecimento é gerado a partir das percepções externas, dos dados e das informações que permitam formular novas concepções. Forma-se a partir dessas premissas o conhecimento científico, o conhecimento tecnológico e de ambos o desenvolvimento de técnicas que aprimoram as atividades humanas. Como o conhecimento é um atributo dinâmico, ficam lançadas as bases para a inovação, ou seja, o permanente aperfeiçoamento das técnicas. Os níveis atuais do conhecimento e das informações passaram a representar um poderoso, senão predominante, fator de produção. As organizações da era informacional, impulsionadas pelos paradigmas da pós-modernidade, se tornaram fontes de geração do conhecimento, principalmente, 
quando se trata de estabelecer estratégias para os objetivos formulados. Há, no contexto, a concepção do conhecimento gerado, e, também da gestão estratégica do conhecimento aplicado aos fins organizacionais. Gera-se, portanto, um conhecimento organizacional cuja gestão é fundamental ao desenvolvimento das atividades em todas as formas de manifestação das atividades sociais e econômicas.

O conhecimento organizacional pode ser gerado a partir de dois pressupostos: o conhecimento tácito, de caráter pessoal, produto dos mapas cognitivos e o conhecimento explícito, o que é passível de transmissão por meio do entendimento e da compreensão. O conhecimento explícito pode formar uma base de dados e informações à estruturação do conhecimento tácito. Nas organizações de modo geral, o conhecimento tácito dá forma a nova universalidade de inovações que fazem avançar o processo de modernidade no campo da produção e do consumo. Seguindo o pensamento de Nonaka e Takeuchi (1997) o conhecimento organizacional é a capacidade de uma empresa de criar um novo conhecimento, difundi-lo na organização como um todo e incorporá-lo a produtos (ou serviços) e sistemas (processos). A partir dessa conceituação pode-se aplicar o conhecimento e a geração de novo conhecimento aos sistemas educacionais e, neles, a determinados processos, como os de avaliação dos cursos a distância.

Nas várias tipologias de organização a geração do conhecimento não é um trabalho isolado, mas representa uma grande interação entre o indivíduo, o grupo, a organização e às relações interorganizacionais. Uma vez criado um conhecimento novo intra-organização, coloca-se, desde logo, a necessidade de se estabelecer uma gestão estratégica, capaz de tornar o novo conhecimento numa ferramenta de desenvolvimento da organização. Toda estratégia é formada por recursos tangíveis e intangíveis, como os recursos tecnológicos e as competências aplicadas a determinado sistema. No campo da educação os recursos e técnicas desenvolvidos e que formam nova gama de conhecimentos, dependem, para a obtenção de resultados positivos, do nível de competência aplicado ao sistema. Ruas (2.001, p.248) destaca três dimensões organizacionais da competência: essenciais, fundamentais à razão da empresa; funcionais, de caráter específico; individuais, de ação gerencial. Essas três dimensões se aplicam a todas as formas de organização, incluindo as educacionais, pois têm significados em relação ao objeto da atividade e à gestão do conhecimento e das competências.

O sistema educacional é lócus de novos conhecimentos, tanto por meio de novas metodologias aplicadas à aprendizagem, como de conhecimento científico e geração de tecnologias avançadas. É um ciclo de conhecimentos que se renova continuamente, criando símbolos e significados que irão influenciar no desenvolvimento de capacitações e, também, na tomada de decisões.

\section{Gestão estratégica do conhecimento na avaliação intitucional em educação a distância}

A avaliação institucional é o processo pelo qual o órgão de educação do sistema de ensino verifica, pondera e atesta a conformidade da instituição de ensino à legislação vigente e aos projetos apresentados em seus planos institucionais. Na literatura encontra-se um consenso acerca da necessidade de avaliação das instituições de ensino e da coerência de procedimentos metodológicos claros que estabeleçam os critérios a serem avaliados. Segundo Freitas e Silva (apud Belloni, 1997, p. 22), "Não se discute mais se avaliação institucional deve ser feita ou não, mas como fazê-la, ou seja, qual a metodologia que conduzirá a maior qualidade e eficiência". É o estudo de novos objetivos e meios à educação (neste estudo a educação a distância); é o que se projeta além da avaliação (Gardner, 1995). Ora o estabelecimento de novos objetivos pressupõe a construção de novos conhecimentos e as estratégias de sua aplicação.

A avaliação Institucional ocupa-se em avaliar as condições gerais de funcionamento dos estabelecimentos de educação superior. A avaliação, realizada pelo INEP, apóia-se na análise de todas as informações relativas à instituição e na verificação in loco, realizada por uma comissão de avaliadores. Um dos principais objetivos da Avaliação Institucional é verificar a execução do Plano de Desenvolvimento Institucional (PDI), que contempla objetivos, metas e ações das instituições de ensino superior. 
É necessário então, adaptar ou criar novas formas de modelagens de informação, ou seja, um novo conhecimento, que permita uma melhor avaliação das Instituições de Ensino Superior, tanto no que diz respeito à avaliação institucional, processo, como de aprendizagem em Educação a Distância.

Avaliação de Processo em Educação Superior a Distância é um dispositivo indispensável à qualificação da aprendizagem em todos os níveis. A avaliação, segundo Durhane (1996, p.40),

não é um procedimento que se restrinja a um momento no tempo ou a um exame. Trata-se, ao contrário, de um processo complexo, diferenciado, permanente e sujeito a um contínuo aperfeiçoamento que se dá em diferentes níveis.

Segundo Rodrigues (1998, p.54), a avaliação é um componente fundamental de qualquer processo ou instituição cujo trabalho seja educação, e ainda complementa que isto se torna ainda mais relevante nos casos de programas de Educação a Distância, diante da falta de um modelo consolidado e de uma tradição no Brasil. Assim, pode-se inferir a importância da construção de novas formas de conhecimento, bem como, geri-las estrategicamente, objetivando a meta maior que é a qualificação do processo de ensino.

A Educação a Distância expressa de forma clara a necessidade do país em garantir uma atualização constante, uma educação continuada que atinja um público novo. Arger (apud Rumble, 2000, p.6), ainda identificou a promessa que a EAD iria atingir as massas por meio do uso das novas tecnologias; suprir uma ampla variedade de necessidades educacionais identificadas, formais e não-formais, atingir grande número de alunos (massificação da educação), elevar os padrões educacionais por meio do uso de materiais de alta qualidade. Esses aspectos se tornam primordiais em função das mudanças sociais e tecnológicas ocorridas na sociedade do conhecimento.

Em 1972, a UNESCO, ao traçar algumas diretrizes para o ensino afirmava que

a educação deve ter por finalidade não apenas formar as pessoas visando uma profissão determinada, mas sobretudo colocá-las em condições de se adaptar a diferentes tarefas e de se aperfeiçoar continuamente, uma vez que as formas de produção e as condições de trabalho evoluem: ela deve tender, assim, a facilitar as reconversões profissionais (UNESCO, 1972)

O Ministério da Educação se mostra preocupado com o tema avaliação na minuta de Decreto elaborada pela Secretaria de Educação a Distância. Nele, no artigo X, é definida a avaliação de cursos e programas como sendo o processo pelo qual o órgão de educação do sistema de ensino verifica, pondera e atesta a conformidade do curso ou programa à legislação vigente, às diretrizes curriculares nacionais definidas pelo Conselho Nacional de Educação e aos respectivos projetos pedagógicos (MEC, 2003).

\section{Método}

Nesse sentido pode-se desenvolver, a partir de uma abordagem teórica, um estudo referido, inicialmente, ao método fenomenológico, objetivando a identificação de padrões e o significado das relações (Creswell, 1994). Segundo Triviños (1987, p. 46) a fenomenologia procura "estabelecer um conhecimento intersubjetivo, de validade geral para todos, isso significa que a fenomenologia estuda o universal, o que é válido para todos os sujeitos".

Por outro lado, a abordagem pode ser do tipo interpretativa, descrita por Gioia e Pitre (1990, p. 591), com as características que seguem:

- objetivos: descrever e explicar para diagnosticar e entender;

- preocupação teórica: converter a construção social da realidade em processo de interpretação; e

- abordagem para a construção teórica: descoberta por meio da análise de códigos. 
A abordagem fenomenológica ou interpretativa justifica-se em função do seu caráter estruturado que procura examinar regularidades e relações que conduzam a princípios universais. Segundo Black (1999, p.27) a condução de um estudo rigoroso implica que o design da pesquisa deve possibilitar que outro pesquisador possa replicar o processo.

Desta forma, pode ser elaborada uma identificação dos processos de avaliação já existentes, bem como um levantamento junto aos atores envolvidos nos cursos para a elaboração de uma modelagem que permita uma avaliação de qualidade, assim como o desenvolvimento de uma métrica única para facilitar o processo.

O estudo a ser elaborado partirá de uma análise da modelagem da informação para a avaliação de cursos oferecidos a distância pelas IES no Brasil. A pertinência do trabalho em relação aos objetivos, aos campos analisados, se justifica pela oferta de subsídios e o estabelecimento de critérios à análise temática. $\mathrm{O}$ resultado será a elaboração de um diagnóstico inicial que permita estabelecer uma visão de planejamento e tomada de decisão no processo de avaliação em educação a distância. Será importante a consideração sistêmica dos processos educacionais, o que permitirá a interatividade entre todas as funções em ativação.

\section{Resultados}

Os estudos a serem desenvolvidos no âmbito da gestão estratégica do conhecimento no campo da avaliação em EAD visam a qualificação do processo ensino-aprendizagem a distância, por meios das tecnologias multimeios. A proposição de especificação de modelo de avaliação integrado para cursos a distância corresponde à integração dos requisitos do MEC, das IES e dos programas das diversas áreas do conhecimento em uma única ferramenta. Possibilita, assim, o acesso rápido às informações confiáveis e pertinentes para cada grupo de agentes no processo de avaliação em EAD.

A informação a ser identificada nos cursos oferecidos a distância compõe um cenário de dados processados tecnicamente e que representam um produto a ser utilizado por usuários, com caráter multifinalitário e multidisciplinar. Estes dados são de origem multidisciplinar, característica da área de conhecimento onde está sendo proposto o estudo, ou seja, na área de educação a distância. Entende-se por multidisciplinaridade a utilização de informação originária de duas ou mais ciências ou setores do conhecimento que conduz a interações reais, a uma certa reciprocidade no intercâmbio, levando a um enriquecimento mútuo. Nesse sentido, a teoria geral dos sistemas compõe com os pressupostos do processo de educação a distância.

O problema a ser pesquisado coloca a questão de como a avaliação em educação a distância contribui para a manutenção de uma educação de qualidade, atendendo os requisitos legais e institucionais. Segundo Ristoff (1999, p. 35) "é uma questão que supera modismos, ela pertence à natureza própria das instituições acadêmicas".

Os estudos a serem desenvolvidos nessa área visam, portanto, alcançar modelagens da informação para a avaliação em educação a distância nas diversas Instituições de Educação Superior. Por meio da modelagem da informação pretende-se facilitar uma visão sistêmica dos cursos a distância (Moore e Kearsley, 1996; Eastmond,1994; Willis, 1996) nas Instituições de Ensino Superior, identificando os cursos com suas estruturas, ambientes de aprendizagem internos, ambientes externos e seus processos críticos. A modelagem da avaliação é um caminho segura à qualificação dos cursos a distância.

\section{Conclusão}

Essa abordagem teórica visa o estudo de modelagem de um sistema de avaliação em cursos de EAD a partir de uma configuração de gestão estratégica do conhecimento. $\mathrm{O}(\mathrm{s})$ sistema(s) possibilitará a análise da informação procedida através da leitura, interpretação e da lógica do modelo. O sistema informatizado utilizará um conjunto de itens, estrategicamente restritivos, que asseguram a racionalização dos procedimentos, produzindo eficiência e o encadeamento lógico necessário para a consolidação dos dados que gerem uma avaliação efetiva dos programas oferecidos a Distância. 
Os fluxos de informação que se estabelecem entre os dados obtidos de diversas fontes, principalmente, das IES e do MEC, permitirão que o sistema desenvolva uma condição analítica. O objetivo é beneficiar toda rede de serviços que utiliza a informação como fundamento para a elaboração de relatórios, pareceres, avaliações e tomada de decisão, bem como para a melhoria da qualidade dos cursos oferecidos a distância.

As novas relações que se estabelecerão entre os atores de pesquisa, as novas relações de produção e trabalho, são componentes de um sistema informacional que ganha expressão a cada ano. A modelagem da informação em um sistema que permita melhorar, aprimorar, de forma integrada a avaliação em cursos oferecidos a distância, é um aspecto relevante quando do desenvolvimento de ações que geram impacto em diversos cenários e agentes. 


\section{Referências bibliograficas}

BELLONI, José Ângelo. Uma metodologia para a avaliação da eficiência produtiva de Universidades Federais Brasileiras. (Doutorado em Engenharia de Produção) - Departamento de Engenharia de Produção, Universidade Federal de Santa Catarina, Florianópolis, 2000.

BLACK, Thomas R. Doing quantitative research in the social sciences: an integrated approach to research design, measurements and statistics. London: SAGE Publications Ltd., 1999.

BRASIL. Ministério de Educação/INEP. Avaliações e estatísticas: avaliação institucional. Disponível em: http://www.inep.gov.br/superior/avaliacao_institucional/. Acesso em: 27 agosto 2003.

CASTELLS, Manuel. A sociedade em rede. São Paulo, Paz e Terra, 1999.

CHAVES, M. Complexidade e transdisciplinaridade: uma abordagem

multidimensional do Setor Saúde. 1998. Disponível em:

http://www.psy.med.br/textos/complexidade/complexidade.pdf $\quad$ Acesso em: 09 abril 2004

CRESWELL, John. Research Design: qualitative and quantitative approaches. SAGE Publications, 1994.

DURHANE, E. Subsídios para a avaliação do ensino superior. Boletim Informativo da CAPES. Brasília, 1996, v.4, n.4, p. 35-40.

EASTM OND, Daniel. Adult learners and internet-based distance education. In: CAHOON, Brad (ed.). Adult learning and the Internet, new directions for adult and continuing education, $\mathrm{n} 78$, summer. 1998.

GARDNER, H. Inteligências múltiplas: a teoria na prática. Porto Alegre: Artes Médicas, 1995.

GILROY, Peter; LONG, Peter; RANGECROFT, Margareth; TRICKER, Tony. Evaluation and the invisible student: theories, practice and problems in evaluating distance education provision. Quality Assurance in Education, v. 9, n. 1, p. 14-22, 2001.

GIOIA, Dennis; PITRE, Evelyn. Multiparadigm perspectives on theory buil ding. Academy of Management Review. v.15, n.4, p.584-602. 1990.

HEEM ANN, V. Avaliação ergonômica de interfaces de bases de dados por meio de checlist especializado. Dissertação (M estrado em Engenharia de Produção) - Departamento de Engenharia de Produção, Universidade Federal de Santa Catarina, Florianópolis, 1997.

MARTENS, Erika; PROSSER, Michael. What constitutes high quality teaching and learning and how to ensure it. Quality Assurance in Education, v. 6, n. 1, p. 28-36, 1998.

MINISTÉRIO DA EDUCAÇÃO. Secretaria de Ensino a Distância. Projeto de Decreto que dispõe sobre a oferta de cursos e programas de educação a distância no âmbito da educação básica e da educação superior, e dá outras providências. Brasília, 2003.

MOORE, Michel G.; KEARSLEY, Greg. Distance education: a systems view. Belmont (USA): Wadsworth Publishing Company, 1996.

MORAES, Marialice de. A monitoria como serviço de apoio ao aluno na educação a distância. (Doutorado em Mídia e Conhecimento) - Departamento de Engenharia de Produção, Universidade Federal de Santa Catarina, Florianópolis, 2004.

NONAKA I. e Takeuchi, H. Criação de conhecimento na empresa. Rio de Janeiro,Campus, 1997

POUNDER, J ames. Institutional performance in higher education: is quality a relevant concept? Quality Assurance in Education, v. 7, n. 3, p. 156-163, 1999.

RISTOFF, Dilvo I. Universidade em foco: reflexões sobre a educação superior. Florianópolis: Insular, 1999.

Rumble, Greville. A Tecnologia da Educação a Distância em Cenários do Terceiro Mundo. In: Preti, Oreste. Educação a Distância. Construindo Significados. Cuiabá: NEAD/IE - UFM T; Brasília, Plano, 2000. URL: http://www.nead.ufmt.br/index.asp?pg=7 Acesso em 21 março 2004.

RODRIGUES, Rosângela S. Modelo de avaliação para cursos no ensino a distância: estrutura, aplicação e avaliação. Dissertação. (Mestrado em Mídia e Conhecimento) - Departamento de Engenharia de Produção, Universidade Federal de Santa Catarina, Florianópolis, 1998.

RUAS, Roberto. Desenvolvimento de competências gerenciais e contribuição da aprendizagem organizacional. In: Gestão estratégica do conhecimento (Org. M aria Tereza Leme Fleury e M oacir de Miranda Oliveira).São Paulo, Atlas, 2001.

TRIVIÑOS, N. S. Introdução à pesquisa em ciências sociais. São Paulo: Atlas, 1987.

VAREY, Richard. (1993). The course for higher education. Managing Service Quality, p. 45-49, Septem

WILLIS, Barry. Distance education at a glance. Idaho, 1996. (Series of Guides prepared by Engineering Outreach at the University of Idaho). Disponível em: http://www.uidaho.edu/evo/distglan.html 